Internat. J. Math. \& Math. Sci.

Vol. 24, No. 5 (2000) 327-334

S0161171200003458

(C) Hindawi Publishing Corp.

\title{
ON A CLASS OF CONTACT RIEMANNIAN MANIFOLDS
}

\author{
JONG TAEK CHO
}

(Received 17 May 1999)

\begin{abstract}
We determine a locally symmetric or a Ricci-parallel contact Riemannian manifold which satisfies a $D$-homothetically invariant condition.

Keywords and phrases. Contact Riemannian manifolds, locally symmetric spaces, Ricciparallel spaces.
\end{abstract}

2000 Mathematics Subject Classification. Primary 53C15, 53C25.

1. Introduction. In [8] Tanno proved that a locally symmetric $K$-contact Riemannian manifold is of constant curvature 1 , which generalizes the corresponding result for a Sasakian manifold due to Okumura [6]. For dimensions greater than or equal to 5 it was proved by Olszak [7] that there are no contact Riemannian structures of constant curvature unless the constant is 1 and in which case the structure is Sasakian. Further, Blair and Sharma [4] proved that a 3-dimensional locally symmetric contact Riemannian manifold is either flat or is Sasakian and of constant curvature 1. By the recent result [5] and private communication with Blair we know that the simply connected covering space of a complete 5-dimensional locally symmetric contact Riemannian manifold is either $S^{5}(1)$ or $E^{3} \times S^{2}(4)$. The question of the classification of locally symmetric contact Riemannian manifolds in higher dimensions is still open.

On the other hand, recently, Blair, Koufogiorgos and Papantoniou [3] introduced a class of contact Riemannian manifolds which is characterized by the equation

$$
R(X, Y) \xi=\kappa(\eta(Y) X-\eta(X) Y)+\mu(\eta(Y) h X-\eta(X) h Y),
$$

where $\kappa, \mu$ are constant and $2 h$ is the Lie derivative of $\phi$ in the direction $\xi$. It is remarkable that this class of spaces is invariant under $D$-homothetic deformations (see [3]). It was also proved in [3] that a Sasakian manifold, in particular, is determined by $\kappa=1$ and further that this class contains the tangent sphere bundle of Riemannian manifolds of constant curvature. In this paper, we determine a locally symmetric or a Ricci-parallel contact Riemannian manifold which satisfies (1.1). More precisely, we prove the following two Theorems 1.1 and 1.2 in Sections 3 and 4.

THEOREM 1.1. Let $M$ be a contact Riemannian manifold satisfying (1.1). Suppose that $M$ is locally symmetric. Then $M$ is the product of flat $(n+1)$-dimensional manifold and an n-dimensional manifold of positive constant curvature equal to 4, or a space of constant curvature 1 and in which case the structure is Sasakian.

THEOREM 1.2. Let $M$ be a contact Riemannian manifold satisfying (1.1). Suppose that $M$ is Ricci-parallel. Then $M$ is the product of flat $(n+1)$-dimensional manifold and 
an $n$-dimensional manifold of positive constant curvature equal to 4 or an EinsteinSasakian manifold.

2. Preliminaries. All manifolds in the present paper are assumed to be connected and of class $C^{\infty}$. A $(2 n+1)$-dimensional manifold $M^{2 n+1}$ is said to be a contact manifold if it admits a global 1-form $\eta$ such that $\eta \wedge(d \eta)^{n} \neq 0$ everywhere. Given a contact form $\eta$, we have a unique vector field $\xi$, which is called the characteristic vector field, satisfying $\eta(\xi)=1$ and $d \eta(\xi, X)=0$ for any vector field $X$. It is well known that there exists an associated Riemannian metric $g$ and a $(1,1)$-type tensor field $\phi$ such that

$$
\eta(X)=g(X, \xi), \quad d \eta(X, Y)=g(X, \phi Y), \quad \phi^{2} X=-X+\eta(X) \xi,
$$

where $X$ and $Y$ are vector fields on $M$. From (2.1) it follows that

$$
\phi \xi=0, \quad \eta \circ \phi=0, \quad g(\phi X, \phi Y)=g(X, Y)-\eta(X) \eta(Y) .
$$

A Riemannian manifold $M$ equipped with structure tensors $(\eta, g)$ satisfying $(2.1)$ is said to be a contact Riemannian manifold and is denoted by $M=(M ; \eta, g)$. Given a contact Riemannian manifold $M$, we define a $(1,1)$-type tensor field $h$ by $h=L_{\xi} \phi / 2$, where $L$ denotes Lie differentiation. Then we may observe that $h$ is symmetric and satisfies

$$
\begin{gathered}
h \xi=0, \quad h \phi=-\phi h, \\
\nabla_{X} \xi=-\phi X-\phi h X,
\end{gathered}
$$

where $\nabla$ is Levi-Civita connection. From (2.3) and (2.4), we see that each trajectory of $\xi$ is a geodesic.

A contact Riemannian manifold for which $\xi$ is Killing is called a $K$-contact Riemannian manifold. It is easy to see that a contact Riemannian manifold is $K$-contact if and only if $h=0$. For a contact Riemannian manifold $M$ one may define naturally an almost complex structure $J$ on $M \times \mathbb{R}$;

$$
J\left(X, f \frac{d}{d t}\right)=\left(\phi X-f \xi, \eta(X) \frac{d}{d t}\right),
$$

where $X$ is a vector field tangent to $M, t$ the coordinate of $\mathbb{R}$, and $f$ a function on $M \times \mathbb{R}$. If the almost complex structure $J$ is integrable, $M$ is said to be normal or Sasakian. It is known that $M$ is normal if and only if $M$ satisfies

$$
[\phi, \phi]+2 d \eta \otimes \xi=0,
$$

where $[\phi, \phi]$ is the Nijenhuis torsion of $\phi$. A Sasakian manifold is characterized by a condition

$$
\left(\nabla_{X} \phi\right) Y=g(X, Y) \xi-\eta(Y) X
$$

for all vector fields $X$ and $Y$ on the manifold. We denote by $R$ the Riemannian curvature tensor of $M$ defined by

$$
R(X, Y) Z=\nabla_{X}\left(\nabla_{Y} Z\right)-\nabla_{Y}\left(\nabla_{X} Z\right)-\nabla_{[X, Y]} Z
$$


for all vector fields $X, Y, Z$ on $M$. It is well known that $M$ is Sasakian if and only if

$$
R(X, Y) \xi=\eta(Y) X-\eta(X) Y
$$

for all vector fields $X$ and $Y$. For a contact Riemannian manifold $M$, the tangent space $T_{p} M$ of $M$ at each point $p \in M$ is decomposed as $T_{p} M=D_{p} \oplus\{\xi\}_{p}$ (direct sum), where we denote $D_{p}=\left\{v \in T_{p} M \mid \eta(v)=0\right\}$. Then $D: p \rightarrow D_{p}$ defines a distribution orthogonal to $\xi$. The $2 n$-dimensional distribution $D$ is called the contact distribution. A contact Riemannian manifold is said to be $\eta$-Einstein if

$$
Q=a I+b \eta \otimes \xi
$$

where $Q$ is the Ricci operator and $a, b$ are smooth functions on $M$.

For more details about the fundamental properties on contact Riemannian manifolds we refer to [1, 2]. Blair [2] proved the following theorem.

THEOREM 2.1. Let $M=(M ; \eta, g)$ be a contact Riemannian manifold and suppose that $R(X, Y) \xi=0$ for all vector fields $X, Y$ on $M$. Then $M$ is locally the product of $(n+1)$-dimensional flat manifold and an $n$-dimensional manifold of positive constant curvature 4.

Recently, Blair, Koufogiorgos, and Papantoniou [3] introduced a class of contact Riemannian manifolds which are characterized by equation (1.1). A $D$-homothetic deformation (cf. [9]) is defined by a change of structure tensors of the form

$$
\bar{\eta}=a \eta, \quad \bar{\xi}=\frac{1}{a} \xi, \quad \bar{\phi}=\phi, \quad \bar{g}=a g+a(a-1) \eta \otimes \eta,
$$

where $a$ is a positive constant. It was shown that [3] a contact Riemannian manifold $M$ satisfying (1.1) is obtained by applying a $D$-homothetic deformation on a contact Riemannian manifold with $R(X, Y) \xi=0$ and that the property (1.1) is invariant under the $D$-homothetic deformation. It is well known that the tangent sphere bundle of a flat Riemannian manifold admits a contact Riemannian structure satisfying $R(X, Y) \xi=0$ [1, page 137]. In [3] the authors classified the 3-dimensional case and showed that this class contains the tangent sphere bundles of Riemannian manifolds of constant sectional curvature. Furthermore in the same paper they showed that $M$ satisfies

$$
\left(\nabla_{Z} h\right) X=(1-\kappa)\{(1-\kappa) g(Z, \phi X)+g(Z, h \phi X)\} \xi+\eta(X)(h \phi+h \phi h) Z-\mu \eta(Z) \phi h X
$$

for any vector fields $X, Z$ on $M$. Here, we state some useful results in [3] to prove our Theorems 1.1 and 1.2.

Proposition 2.2. Let $M=(M ; \eta, g)$ be a contact Riemannian manifold which satisfies (1.1), where $\kappa<1$.

(i) If $X, Y \in D(\lambda)$ (respectively, $D(-\lambda)$ ), then $\nabla_{X} Y \in D(\lambda)$ (respectively, $D(-\lambda)$ ).

(ii) If $X \in D(\lambda), Y \in D(-\lambda)$, then $\nabla_{X} Y$ (respectively, $\left.\nabla_{Y} X\right) \in D(-\lambda) \oplus D(0)$ (respectively, $D(\lambda) \oplus D(0)$ ) . 
THEOREM 2.3. Let $M=(M ; \eta, g)$ be a contact Riemannian manifold which satisfies (1.1), then $\kappa \leq 1$. If $\kappa=1$, then $h=0$ and $M$ is a Sasakian manifold. If $k<1$, then $M$ admits three mutually orthogonal and integrable distributions $D(0), D(\lambda)$, and $D(-\lambda)$, defined by the eigenspaces of $h$, where $\lambda=\sqrt{1-\kappa}$. Moreover

$$
\begin{aligned}
& R\left(X_{\lambda}, Y_{\lambda}\right) Z_{-\lambda}=(\kappa-\mu)\left\{g\left(\phi Y_{\lambda}, Z_{-\lambda}\right) \phi X_{\lambda}-g\left(\phi X_{\lambda}, Z_{-\lambda}\right) \phi Y_{\lambda}\right\}, \\
& R\left(X_{-\lambda}, Y_{-\lambda}\right) Z_{\lambda}=(\kappa-\mu)\left\{g\left(\phi Y_{-\lambda}, Z_{\lambda}\right) \phi X_{-\lambda}-g\left(\phi X_{-\lambda}, Z_{\lambda}\right) \phi Y_{-\lambda}\right\}, \\
& R\left(X_{\lambda}, Y_{-\lambda}\right) Z_{-\lambda}=\kappa g\left(\phi X_{\lambda}, Z_{-\lambda}\right) \phi Y_{-\lambda}+\mu g\left(\phi X_{\lambda}, Y_{-\lambda}\right) \phi Z_{-\lambda}, \\
& R\left(X_{\lambda}, Y_{-\lambda}\right) Z_{\lambda}=-\kappa g\left(\phi Y_{-\lambda}, Z_{\lambda}\right) \phi X_{\lambda}-\mu g\left(\phi Y_{-\lambda}, X_{\lambda}\right) \phi Z_{\lambda}, \\
& R\left(X_{\lambda}, Y_{\lambda}\right) Z_{\lambda}=\{2(1+\lambda)-\mu\}\left\{g\left(Y_{\lambda}, Z_{\lambda}\right) X_{\lambda}-g\left(X_{\lambda}, Z_{\lambda}\right) Y_{\lambda}\right\}, \\
& R\left(X_{-\lambda}, Y_{-\lambda}\right) Z_{-\lambda}=\{2(1-\lambda)-\mu\}\left\{g\left(Y_{-\lambda}, Z_{-\lambda}\right) X_{-\lambda}-g\left(X_{-\lambda}, Z_{-\lambda}\right) Y_{-\lambda}\right\},
\end{aligned}
$$

where $X_{\lambda}, Y_{\lambda}, Z_{\lambda} \in D(\lambda)$ and $X_{-\lambda}, Y_{-\lambda}, Z_{-\lambda} \in D(-\lambda)$.

THEOREM 2.4. For a contact Riemannian manifold satisfying (1.1) with $\kappa<1$, the Ricci operator $Q$ is given by

$$
Q=\{2(n-1)-n \mu\} I+\{2(n-1)+\mu\} h+\{2(1-n)+n(2 \kappa+\mu)\} \eta \otimes \xi .
$$

For more results about a contact Riemannian manifold satisfying (1.1), we refer to [3].

3. Proof of Theorem 1.1. Let $M^{2 n+1}$ be a $(2 n+1)$-dimensional contact Riemannian manifold which satisfies (1.1). Suppose that $M$ is locally symmetric, that is, $\nabla R=0$. In view of the results of the Sasakian case [6] and the 3-dimensional contact Riemannian case [4], we now assume that $n>1$ and $M$ is non-Sasakian $(\kappa \neq 1)$. From $h \xi=0$, with (2.4) we have

$$
\left(\nabla_{Z} h\right) \xi=\nabla_{Z}(h \xi)-h \nabla_{Z} \xi=(h \phi+h \phi h) Z .
$$

If we differentiate (1.1) covariantly, then using (2.4) we get

$$
\begin{aligned}
R(X, Y)(-\phi Z-\phi h Z)= & \kappa\{g(-\phi Z-\phi h Z, Y) X-g(-\phi Z-\phi h Z, X) Y\} \\
+ & \mu\left\{g(-\phi Z-\phi h Z, Y) h X+\eta(Y)\left(\nabla_{Z} h\right) X\right. \\
& \left.-g(-\phi Z-\phi h Z, X) h Y-\eta(X)\left(\nabla_{Z} h\right) Y\right\}
\end{aligned}
$$

for any vector fields $X, Y$ on $M$. Putting $Y=\xi$, then with (2.2), (2.3), and (3.1) we have

$$
R(X, \xi)(-\phi Z-\phi h Z)=\kappa g(\phi Z+\phi h Z, X) \xi+\mu\left\{\left(\nabla_{Z} h\right) X-\eta(X)(h \phi+h \phi h) Z\right\} .
$$

Together with (1.1) we have

$$
\mu\left(\nabla_{Z} h\right) X=\mu\{\eta(X)(h \phi+h \phi h) Z+g((h \phi+h \phi h) Z, X) \xi\} .
$$

From (2.12) and (3.4) we have

$$
\begin{aligned}
& \mu\{\eta(X)(h \phi+h \phi h) Z+g((h \phi+h \phi h) Z, X) \xi\} \\
& \quad=\mu\{(1-\kappa)\{(1-\kappa) g(Z, \phi X)+g(Z, h \phi X)\} \xi+\eta(X)(h \phi+h \phi h) Z-\mu \eta(Z) \phi h X\}
\end{aligned}
$$


for any vector fields $X, Z$ in $M$. If we put $Z=\xi$, then we have

$$
\mu^{2} \phi h X=0
$$

Since $M$ is not Sasakian, we have $\mu=0$. Now, we consider the following equation in Theorem 2.3:

$$
R\left(X_{\lambda}, Y_{\lambda}\right) Z_{\lambda}=2(1+\lambda)\left\{g\left(Y_{\lambda}, Z_{\lambda}\right) X_{\lambda}-g\left(X_{\lambda}, Z_{\lambda}\right) Y_{\lambda}\right\}
$$

where $X_{\lambda}, Y_{\lambda}, Z_{\lambda} \in D(\lambda)$. Differentiating (3.7) covariantly with respect to $V_{-\lambda} \in D(-\lambda)$, then since $M$ is locally symmetric we have

$$
\begin{aligned}
R\left(\nabla_{V_{-\lambda}} X_{\lambda}, Y_{\lambda}\right) & Z_{\lambda}+R\left(X_{\lambda}, \nabla_{V_{-\lambda}} Y_{\lambda}\right) Z_{\lambda}+R\left(X_{\lambda}, Y_{\lambda}\right) \nabla_{V_{-\lambda}} Z_{\lambda} \\
=2(1+\lambda)\{ & g\left(\nabla_{V_{-\lambda}} Y_{\lambda}, Z_{\lambda}\right) X_{\lambda}+g\left(Y_{\lambda}, \nabla_{V_{-\lambda}} Z_{\lambda}\right) X_{\lambda}+g\left(Y_{\lambda}, Z_{\lambda}\right) \nabla_{V_{-\lambda}} X_{\lambda} \\
& \left.-g\left(\nabla_{V_{-\lambda}} X_{\lambda}, Z_{\lambda}\right) Y_{\lambda}-g\left(X_{\lambda}, \nabla_{V_{-\lambda}} Z_{\lambda}\right) Y_{\lambda}-g\left(X_{\lambda}, Z_{\lambda}\right) \nabla_{V_{-\lambda}} Y_{\lambda}\right\} .
\end{aligned}
$$

Together with Proposition 2.2 and using (3.7) again we get

$$
\begin{array}{r}
g\left(\nabla_{V_{-\lambda}} X_{\lambda}, \xi\right) R\left(\xi, Y_{\lambda}\right) Z_{\lambda}+g\left(\nabla_{V_{-\lambda}} Y_{\lambda}, \xi\right) R\left(X_{\lambda}, \xi\right) Z_{\lambda}+g\left(\nabla_{V_{-\lambda}} Z_{\lambda}, \xi\right) R\left(X_{\lambda}, Y_{\lambda}\right) \xi \\
=2(1+\lambda)\left\{g\left(Y_{\lambda}, Z_{\lambda}\right) g\left(\nabla_{V_{-\lambda}} X_{\lambda}, \xi\right) \xi-g\left(X_{\lambda}, Z_{\lambda}\right) g\left(\nabla_{V_{-\lambda}} Y_{\lambda}, \xi\right) \xi\right\} .
\end{array}
$$

From (1.1), by using the property of the curvature tensor, we get

$$
R(\xi, X) Y=\kappa(g(Y, X) \xi-\eta(Y) X)+\mu(g(h Y, X) \xi-\eta(Y) h X) .
$$

By using (1.1), (2.1), and (3.10) we have

$$
(\kappa-2 \lambda-2)\left\{g\left(Y_{\lambda}, Z_{\lambda}\right) g\left(X_{\lambda}, \phi V_{-\lambda}+\phi h V_{-\lambda}\right) \xi-g\left(X_{\lambda}, Z_{\lambda}\right) g\left(Y_{\lambda}, \phi V_{-\lambda}+\phi h V_{-\lambda}\right) \xi\right\}=0,
$$

and thus we have

$$
(1-\lambda)(\kappa-2 \lambda-2)\left\{g\left(Y_{\lambda}, Z_{\lambda}\right) g\left(X_{\lambda}, \phi V_{-\lambda}\right) \xi-g\left(X_{\lambda}, Z_{\lambda}\right) g\left(Y_{\lambda}, \phi V_{-\lambda}\right) \xi\right\}=0 .
$$

We may take an adapted orthonormal basis $\left\{\xi, e_{i}, \phi e_{i}\right\}$ such that $h \xi=0, h e_{i}=\lambda_{i} e_{i}$ and $h \phi e_{i}=-\lambda_{i} \phi e_{i}, i=1,2, \ldots, n$ at any point $p \in M$. Since $g\left(\phi e_{i}, \phi V_{-\lambda}\right)=0$ and $g\left(Y_{\lambda}, \xi\right) g\left(\xi, \phi V_{-\lambda}\right)=0$, from (3.12) we have

$$
\begin{aligned}
(1-\lambda)(\kappa-2 \lambda-2)\{ & \sum_{1}^{n} g\left(Y_{\lambda}, e_{i}\right) g\left(e_{i}, \phi V_{-\lambda}\right) \xi \\
& +\sum_{1}^{n} g\left(Y_{\lambda}, \phi e_{i}\right) g\left(\phi e_{i}, \phi V_{-\lambda}\right) \xi+g\left(Y_{\lambda}, \xi\right) g\left(\xi, \phi V_{-\lambda}\right) \xi \\
& \left.-\sum_{1}^{n} g\left(e_{i}, e_{i}\right) g\left(Y_{\lambda}, \phi V_{-\lambda}\right) \xi\right\}=0 .
\end{aligned}
$$

And hence, we obtain

$$
(1-n)(1-\lambda)(\kappa-2 \lambda-2) g\left(Y_{\lambda}, \phi V_{-\lambda}\right) \xi=0 .
$$


If we put $\phi V_{-\lambda}=Y_{\lambda}$ in (3.14), then it follows that

$$
(1-n)(1-\lambda)(\kappa-2 \lambda-2)=0,
$$

where $X, Y$ are vector fields on $M$. Since $n>1$ and $\kappa=1-\lambda^{2}$, we conclude that $\kappa=$ $\mu=0$, that is, $M$ satisfies $R(X, Y) \xi=0$ for any vector fields $X, Y$ in $M$. Therefore by the results in $[4,6]$ and Theorem 2.1 we have proved Theorem 1.1.

4. Proof of Theorem 1.2. Let $M$ be a contact Riemannian manifold which satisfies (1.1). Suppose that $M$ is Ricci-parallel, that is, $\nabla Q=0$. From (1.1) and (2.3) we have

$$
Q \xi=2 n \kappa \xi
$$

From (2.4) and (4.1), we have

$$
\left(\nabla_{Z} Q\right) \xi=-2 n \kappa(\phi+\phi h) Z+Q(\phi+\phi h) Z .
$$

Since $M$ is Ricci-parallel, we have

$$
Q(\phi+\phi h) Z=2 n \kappa(\phi+\phi h) Z
$$

for any vector field $Z$ on $M$. If we substitute $Z$ with $\phi Z$, then by using (2.1) and (4.1), we obtain that

$$
Q(I-h)=2 n \kappa(I-h)
$$

If $\kappa=1(h \equiv 0)$, then from (4.4) we see that $M$ is Einstein-Sasakian and the scalar curvature $\tau=2 n(2 n+1)$.

Now, we assume that $\kappa \neq 1$, that is, $M$ is non-Sasakian. Differentiating (2.14) covariantly, then it follows that

$$
\begin{aligned}
\left(\nabla_{Z} Q\right) X= & \{2(n-1)+\mu\}\left(\nabla_{Z} h\right) X-\{2(1-n)+n(2 \kappa+\mu)\} g((\phi+\phi h) Z, X) \xi \\
& -\{2(1-n)+n(2 \kappa+\mu)\} \eta(X)(\phi+\phi h) Z,
\end{aligned}
$$

and thus we get

$\{2(n-1)+\mu\}\left(\nabla_{Z} h\right) X=\{2(1-n)+n(2 \kappa+\mu)\}\{g((\phi+\phi h) Z, X) \xi+\eta(X)(\phi+\phi h) Z\}$.

Together with (2.12) we have

$$
\begin{array}{r}
\{2(n-1)+\mu\}[(1-\kappa)\{(1-\kappa) g(Z, \phi X)+g(Z, h \phi X)\} \xi+\eta(X)(h \phi+h \phi h) Z-\mu \eta(Z) \phi h X] \\
=\{2(1-n)+n(2 \kappa+\mu)\}\{g((\phi+\phi h) Z, X) \xi+\eta(X)(\phi+\phi h) Z\} .
\end{array}
$$

If we put $Z=\xi$ in (4.7), then we have

$$
\mu\{2(n-1)+\mu\} \phi h=0,
$$


and hence we see that $\mu=0$ or $2(n-1)+\mu=0$. Now, we discuss our arguments divided into two cases: (i) $\mu=0$, (ii) $2(n-1)+\mu=0$.

The case (i) $\mu=0$. Then (4.7) becomes

$$
\begin{array}{r}
2(n-1)[(1-\kappa)\{(1-\kappa) g(Z, \phi X)+g(Z, h \phi X)\} \xi+\eta(X)(h \phi+h \phi h) Z] \\
=\{2(1-n)+2 n \kappa\}\{g((\phi+\phi h) Z, X) \xi+\eta(X)(\phi+\phi h) Z\} .
\end{array}
$$

Putting $X=\xi$, then by using (2.2) and (2.3) we get

$$
2(1-n)\left(\phi h+\phi h^{2}\right) Z=\{2(1-n)+2 n \kappa\}(\phi+\phi h) Z .
$$

We apply $\phi$ and use (2.2), then we have

$$
2(n-1) h^{2} Z+2 n \kappa h Z+\{2(1-n)+2 n \kappa\}(Z-\eta(Z) \xi)=0 .
$$

Since the trace of $h^{2}=2 n(1-\kappa)$ and the trace of $h=0$, we have $\kappa=0$. Thus, $M$ satisfies $R(X, Y) \xi=0$. By Theorem 2.1 we conclude that $M$ is locally the product of $(n+1)$-dimensional manifold and an $n$-dimensional manifold of positive constant curvature 4 .

The case (ii) $2(n-1)+\mu=0$. Then (2.14) is reduced to

$$
Q=\{2(n-1)-n \mu\} I+\{2(1-n)+n(2 \kappa+\mu)\} \eta \otimes \xi
$$

that is, $M$ is $\eta$-Einstein. From (4.7) we get

$$
\{2(1-n)+n(2 \kappa+\mu)\}\{g(-(\phi+\phi h) Z, X) \xi+\eta(X)(\phi+\phi h) Z\}=0
$$

for any vector field $X, Z$ on $M$. Putting $X=\xi$ in (4.13), then we have

$$
\{2(1-n)+n(2 \kappa+\mu)\}(\phi+\phi h) Z=0 .
$$

If $2(1-n)+n(2 \kappa+\mu)=0$, since $\mu=2(1-n)$ we have

$$
\kappa=\frac{n^{2}-1}{n}
$$

But we know that $\kappa<1$, and thus we see that $n$ must be equal to 1 and hence $\kappa=\mu=0$. Otherwise, $2(1-n)+n(2 \kappa+\mu) \neq 0$, then (4.14) becomes

$$
\phi+\phi h=0
$$

which is impossible. Therefore, summing up all the arguments in this section we have Theorem 1.2.

REMARK 4.1. $\mathbb{R}^{3}\left(x^{1}, x^{2}, x^{3}\right)$ or $T^{3}$ (torus) with $\eta=1 / 2\left(\cos x^{3} d x^{1}+\sin x^{3} d x^{2}\right)$ and $g_{i j}=1 / 4 \delta_{i j}$ is an $\eta$-Einstein, non-Sasakian, contact Riemannian manifold (cf. [1]). 
ACKNOWLEDGEMENT. This study was financially supported by Chonnam National University in the program, 1999 and in part by BSRI 98-1425.

\section{REFERENCES}

[1] D. E. Blair, Contact Manifolds in Riemannian Geometry, Lecture Notes in Mathematics, Vol. 509, Springer-Verlag, Berlin, New York, 1976. MR 57\#7444. Zbl 319.53026.

[2] _ _ Two remarks on contact metric structures, Tôhoku Math. J. (2) 29 (1977), no. 3, 319-324. MR 57\#4043. Zbl 376.53021.

[3] D. E. Blair, T. Koufogiorgos, and B. J. Papantoniou, Contact metric manifolds satisfying a nullity condition, Israel J. Math. 91 (1995), no. 1-3, 189-214. MR 96f:53037. Zbl 837.53038.

[4] D. E. Blair and R. Sharma, Three-dimensional locally symmetric contact metric manifolds, Boll. Un. Mat. Ital. A (7) 4 (1990), no. 3, 385-390. MR 92d:53030. Zbl 721.53046.

[5] D. E. Blair and J. M. Sierra, 5-dimensional locally symmetric contact metric manifolds, Boll. Un. Mat. Ital. A (7) 7 (1993), no. 2, 299-311. MR 94h:53039. Zbl 811.53029.

[6] M. Okumura, Some remarks on space with a certain contact structure, Tôhoku Math. J. (2) 14 (1962), 135-145. MR 26\#708. Zbl 119.37701.

[7] Z. Olszak, On contact metric manifolds, Tôhoku Math. J. (2) 31 (1979), no. 2, 247-253. MR 81b:53032. Zbl 403.53018.

[8] S. Tanno, Locally symmetric K-contact Riemannian manifolds, Proc. Japan Acad. 43 (1967), 581-583. MR 37\#861. Zbl 155.49802.

[9] _ The topology of contact Riemannian manifolds, Illinois J. Math. 12 (1968), 700-717. MR 38\#2803. Zbl 165.24703.

Jong Taek Cho: Department of Mathematics, Chonnam National University, KWANGJU 500-757, KOREA

E-mail address: jtcho@chonnam.chonnam.ac.kr 


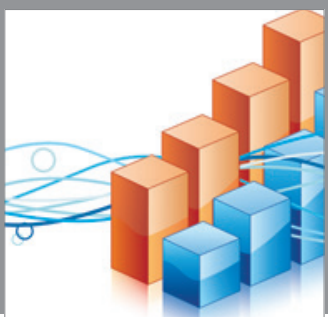

Advances in

Operations Research

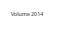

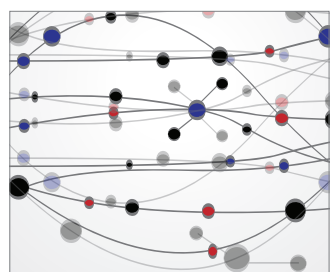

\section{The Scientific} World Journal
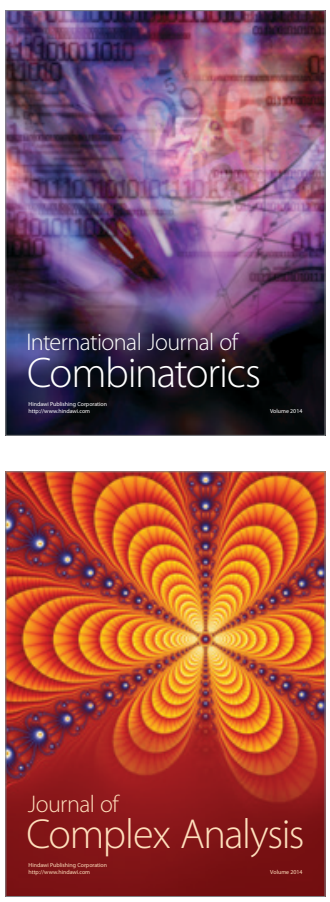

International Journal of

Mathematics and

Mathematical

Sciences
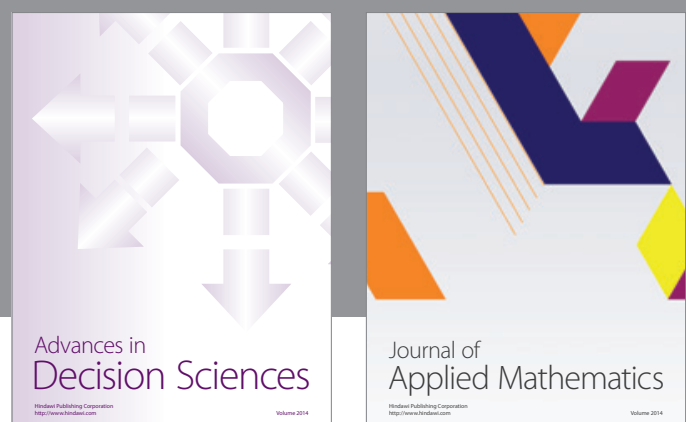

Journal of

Applied Mathematics
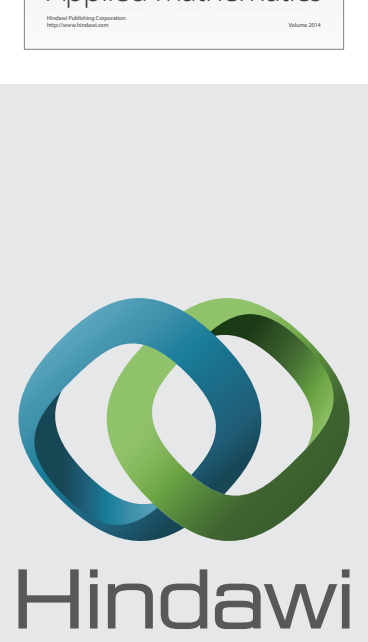

Submit your manuscripts at http://www.hindawi.com
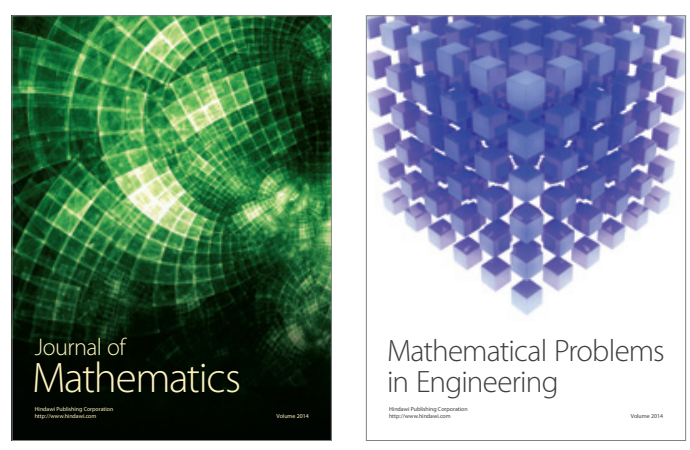

Mathematical Problems in Engineering
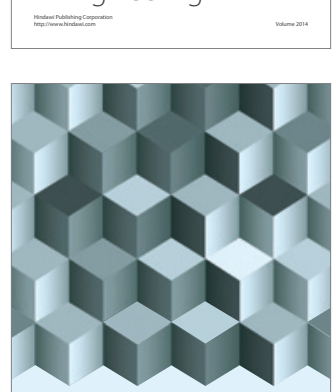

Journal of

Function Spaces
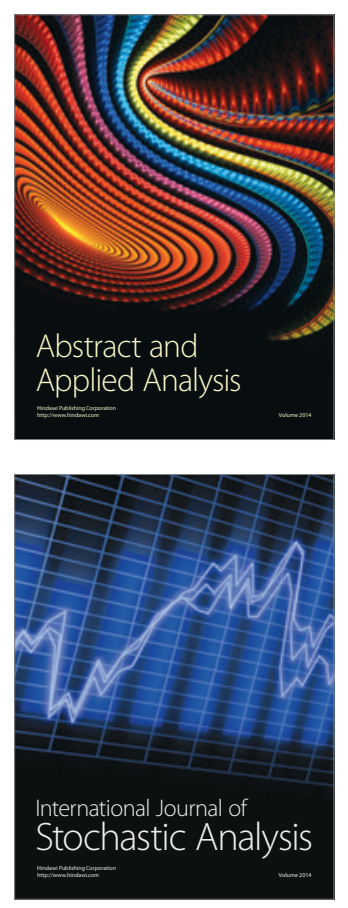

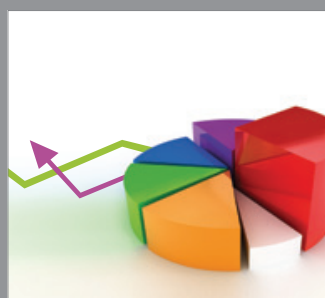

ournal of

Probability and Statistics

Promensencen
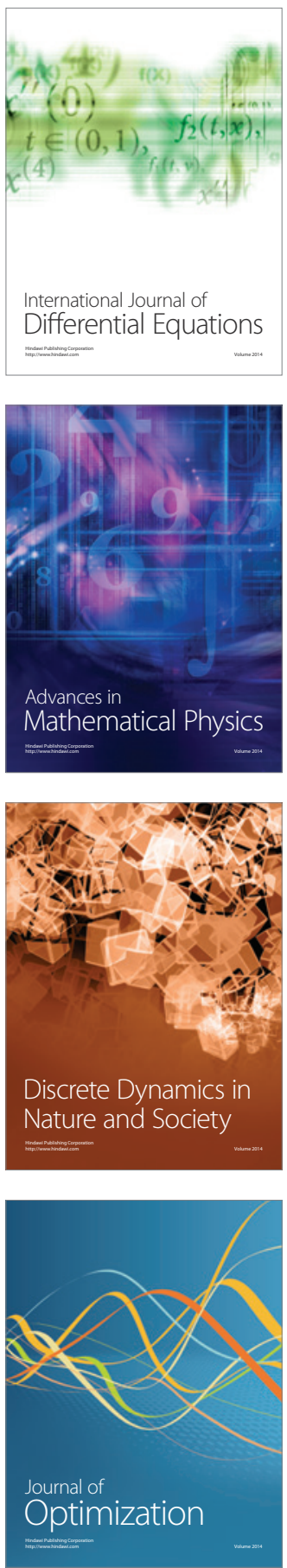